\title{
Modeling and predicting individual tacit coordination ability
}

\author{
Dor Mizrahi* ${ }^{*}$, Ilan Laufer and Inon Zuckerman
}

\begin{abstract}
Background: Previous experiments in tacit coordination games hinted that some people are more successful in achieving coordination than others, although the variability in this ability has not yet been examined before. With that in mind, the overarching aim of our study is to model and describe the variability in human decision-making behavior in the context of tacit coordination games.

Methods: In this study, we conducted a large-scale experiment to collect behavioral data, characterized the distribution of tacit coordination ability, and modeled the decision-making behavior of players. First, we measured the multimodality in the data and described it by using a Gaussian mixture model. Then, using multivariate linear regression and dimensionality reduction (PCA), we have constructed a model linking between individual strategic profiles of players and their coordination ability. Finally, we validated the predictive performance of the model by using external validation.

Results: We demonstrated that coordination ability is best described by a multimodal distribution corresponding to the levels of coordination ability and that there is a significant relationship between the player's strategic profile and their coordination ability. External validation determined that our predictive model is robust.

Conclusions: The study provides insight into the amount of variability that exists in individual tacit coordination ability as well as in individual strategic profiles and shows that both are quite diverse. Our findings may facilitate the construction of improved algorithms for human-machine interaction in diverse contexts. Additional avenues for future research are discussed.
\end{abstract}

Keywords: Tacit coordination, Decision-making, Cognitive modeling

\section{Background}

A tacit coordination game is one in which two individuals are rewarded for making the same choice from the same set of alternatives, and any form of communication between the players is not allowed or not possible (e.g., $[1-4])$. Such problems have been formally modeled in game theory as games with multiple Nash equilibria solutions with equal values [5]. Tacit coordination problems can be found in real-life situations such as tacit collusion among retail chains [6], social coordination, allowing, for

*Correspondence: dor.mizrahi1@msmail.ariel.ac.il

Department of Industrial Engineering and Management, Ariel University, Ariel, Israel example, the convergence on a new technological platform [7], and auction-based collaboration scenarios [8].

Since Schelling's seminal work in 1960 [4] many experiments have shown that people somehow manage to converge on a solution more effectively than what was predicted by the game-theoretical analysis. Apparently, for different reasons, some equilibria solutions appear more prominent than others for the players in the game. These solutions are denoted as focal points. In contrast, the game-theoretical framework fails to explain people's decisions in such games [1], mostly because the problem of deciding between multiple Nash equilibria, which is one of the major challenges

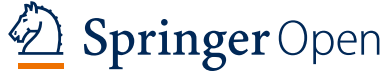

(c) The Author(s) 2022. Open Access This article is licensed under a Creative Commons Attribution 4.0 International License, which permits use, sharing, adaptation, distribution and reproduction in any medium or format, as long as you give appropriate credit to the original author(s) and the source, provide a link to the Creative Commons licence, and indicate if changes were made. The images or other third party material in this article are included in the article's Creative Commons licence, unless indicated otherwise in a credit line to the material. If material is not included in the article's Creative Commons licence and your intended use is not permitted by statutory regulation or exceeds the permitted use, you will need to obtain permission directly from the copyright holder. To view a copy of this licence, visit http://creativecommons.org/licenses/by/4.0/. 
of game theory $[4,9] .{ }^{1}$ However, as Schelling showed in his experiments players do manage to converge on a same solution by relying on salient labels that help distinguish a set of more prominent solutions, which are the focal points $[10,11]$. Thus, game theory lacks the ability to capture cognitive heuristics that people apply when solving coordination problem, such as symmetry, proximity, or extremeness $[12,13]$. Therefore, experiments in behavioral game theory try to fill this gap by constructing cognitive models describing decision-making heuristics (e.g., [14-18]).

Another question that has been left unanswered is the degree of heterogeneity in peoples' ability to successfully coordinate in tacit coordination games. In other words, given a set of tacit coordination problems, it seems that some people manage to successfully coordinate most of their answers with the unknown partner, while others experience difficulties in doing so. This ability to succeed in tacit coordination tasks was hinted at by Bacharach in [1] and was named "Schelling's competence". In other words, people who manage to coordinate most of their answers are regarded as having high Schelling's competence. The variation in the level of Schelling's competence might be explained by the propensity of applying multiple salient selection rules. The weighted combination of the selection rules was denoted as a strategic profile $[15,16,19]$. Each strategic profile reflects the subjective preferences of individual players regarding a set of heuristics or prominent and salient selection rules $[15,16,19]$. Thus, a strategic profile may be regarded as a weighted combination of the different selection rules utilized by a specific player across different game instances to achieve a successful coordination.

The overarching aim of our study was to model the relationship between individual coordination ability and decision-making within the framework of a tacit coordination game. To that end, we have conducted a large-scale tacit coordination experiment to collect behavioral data. We have first described the variability in individual coordination ability (iCA) [16, 17] among the players, detected the predominant selection rules (e.g., spatial proximity) that players have utilized, and finally modeled the relationship between iCA and the individual strategic profiles [16].

Next, we have validated the proposed model by predicting the individual coordination ability of a player based on their constructed strategic profile. Understanding the differences in individual's tacit coordination abilities as well as their unique strategic

\footnotetext{
${ }^{1}$ Quoting Binmore ([61], p. 262), "The equilibrium selection problem is perhaps the greatest challenge facing modern game theory".
}

profiles will allow a better prediction of human behavior in tacit coordination scenarios and consequently the improvement of algorithms for human-machine interactions.

\section{Methods}

\subsection{Experimental design}

To test the coordination ability of players we used the "Assign Circles" [12, 13, 15, 20] tacit coordination game. The players were presented with 14 different "Assign Circles" decision problems, each associated with a different board layout: 10 predefined problems were taken from [12] and additional 4 were randomly generated layouts. To avoid consistent bias that may have been caused by the order of the games, the games were presented randomly for each of the players. In each of the decision problems the players were asked to assign circles to squares with the aim of coordinating their assignment with an unknown player, who was presented with the same board layout (see Fig. 1 for a game example). That is, a successful coordination was achieved when both players attached all the circles to the same squares. In case of a successful coordination both players gained a point and each player could accumulate additional points as the game progressed. Both players had no communication capability at all, and their results were only revealed after all the games had been completed.

Based on $[12,13,15,16]$ it was assumed that participants would utilize three main selection rules when playing the "Assign Circles" games, as follows: (1) closeness-assigning each circle to the closest available square; (2) accession-assigning circles which are close to one another to the same square; and (3) equality-assigning the same number of circles to each of the two available squares. We examined the variability in how these three rules were weighted and aggregated as part of the decision-making process of each individual participant, for the "Assign Circles" game. Importantly, we do not claim that these three, domain dependent rules, represent an exhaustive listing of all the rules that are available for this game (see [12, 16, 18, 21-23]). Rather, we merely suggest that these three prominent selection rules should provide enough variability in the individual strategic profiles.

As the first ten decision problems of the game have a fixed layout, we can analyze each one of them using the above mentioned selection rules and detect the expected solution by implementing each of the rules in each game. In this way, we may potentially predict the solutions by which the players will choose to establish a focal point had we known the strategic profile of each participant and assuming consistency between games. For example, let us examine the game presented in Fig. 1. First, it is evident that the equality rule is not applicable as there 


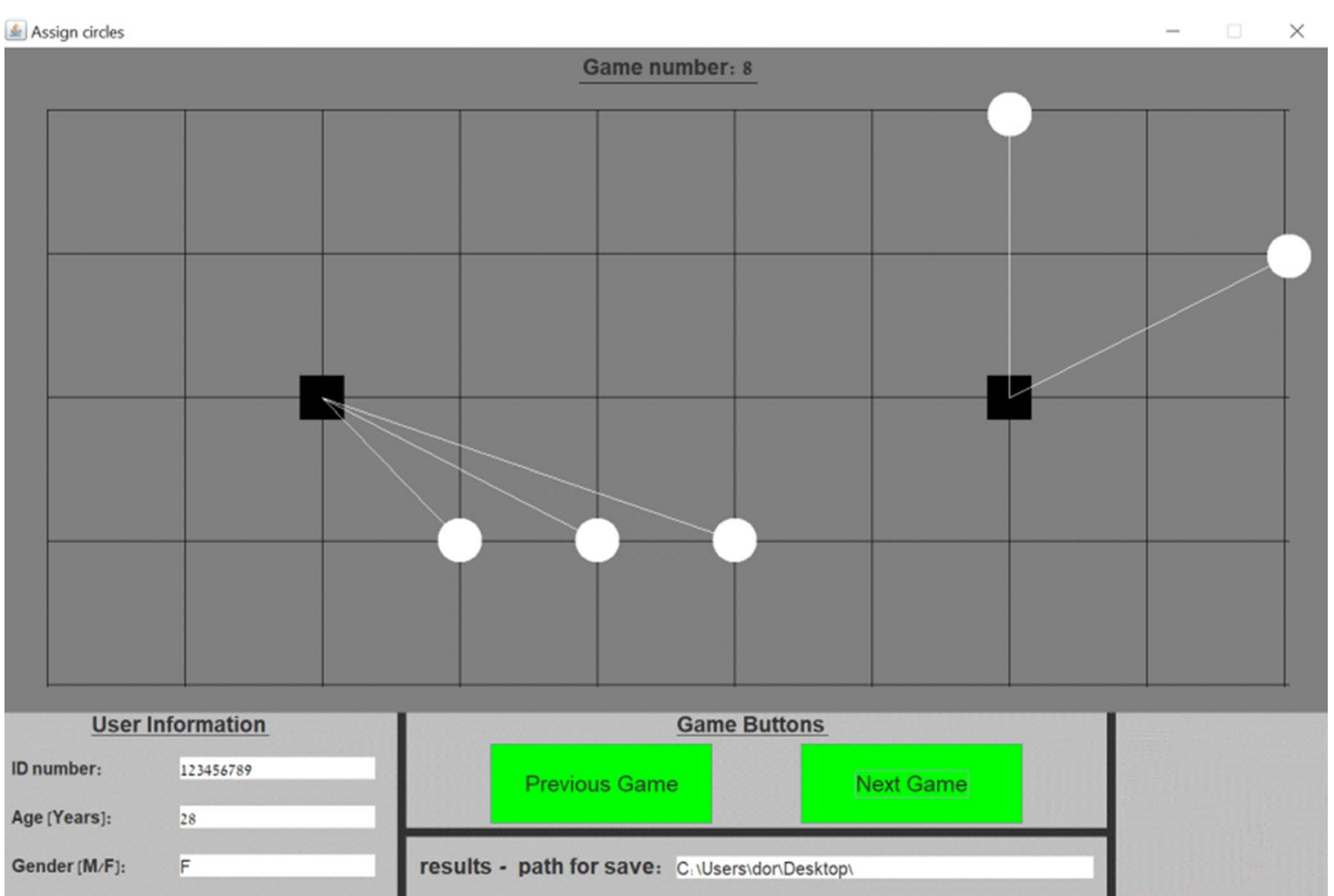

Fig. 1 "Assign Circles" application window

are 5 circles and any division of either $2-3$ or 3-2 will not enable the utilization of this rule. Second, the accession rule solves the coordination problem as illustrated in Fig. 1, which displays two distinct groups of circles. Lastly, using the closeness rule we expect the middle circle to be connected to the right-hand square rather than to the left one as displayed in Fig. 1. In a similar manner we can describe the resultant solution attained by using each of the selection rules for each game instance.

\subsection{Participants}

The participants were 93 students that were enrolled in one of the courses on campus [49 of whom were female, mean age $=22.93$ (years), SD $=1.97$ ]. The 93 participants were randomly assigned to two consecutive sessions, with 48, and 45 participants in each of the sessions, respectively. All students were seated in one class and each student sat in front of a desktop monitor. Before the onset of the experiment, participants received an explanation regarding the overarching aim of the study, the experimental procedure, and the graphics of the application window. As participants were rewarded according to their performance on the "Assign Circles" task, they have also received an explanation regarding the criteria for allocation of rewards (in the form of course credit) according to how many points they gained. To verify that participants understood the concept of coordination, they were given several training examples by presenting screenshots of different layouts of discs for each of the players. Moreover, prior to the start of the session, several questions were asked by the experimenter regarding the usage of the application to ensure that participants know how to operate it.

The study was approved by the IRB committee of Ariel University. All participants provided verbal informed consent for the experiment.

\subsection{Measures}

\subsubsection{Coordination index $(\mathrm{Cl})$}

The CI measure proposed by Mehta et al. [12] is a statistical measure that allows determining the difficulty of coordination in a specific game. Specifically, the higher the $\mathrm{CI}$, the easier it is to coordinate between two random players. Consider a coordination game with the set of possible solutions $L=\left\{l_{1}, \ldots, l_{n}\right\}$, where a solution is an implementation of specific strategies, and $N$ is the number players, when each of them plays the game only once with an unknown anonymous partner. For each solution $l_{j}$ let $m_{j}$ be the number of individuals who choose it, then the coordination index $\mathrm{c}$ is given by: 


$$
c=\frac{\sum_{j} m_{j}\left(m_{j}-1\right)}{N(N-1)} .
$$

This index measures the probability that two distinct individuals, chosen at random without replacement from the set of $\mathrm{N}$ individuals, choose the same solution. It takes the value 1 if all individuals selected the same single solution and 0 if everyone selected a different solution. If solutions are chosen at random, the expected value of the index is $\frac{1}{n}$. of choosing each of the abovementioned strategies by a single player, we first defined the strategy rate (SR). SR is defined as the probability that a specific player will choose using one of the three selection rules in one of the decision problems included in the Assign Circles game. The SR was calculated based on the behavioral performance data of each player in each decision problem. To compute the SR measure, we first defined a Game Tag (GT) variable for each strategy, which can have one of three different values as follows:

$$
\mathrm{GT}(i, k)_{\text {strategy }}=\left\{\begin{array}{l}
\mathbf{1}, \text { if the strategy was available in the } k \text { th game and the } i \text { th player used it } \\
\mathbf{0}, \text { if the strategy was not available in the } k \text { th game } \\
-\mathbf{1}, \text { if the strategy was available in the } k \text { th game and the } i \text { th player did not use it }
\end{array} .\right.
$$

\subsubsection{Individual coordination ability (iCA)}

To measure the coordination ability of each player, we assessed their ability to coordinate with all 92 other participants in the experiment rather than with a single random participant. For this purpose, we calculated the total number of games in which each player was able to coordinate their responses against the entire experimental population and normalized it by the total number of games. It should be noted that the calculation was only carried out on the ten predefined games, but not on the four randomized games, since only the predefined games were kept constant across participants. iCA $[16,17,24]$ is formally defined as follows:

$$
\mathrm{iCA}(i)=\sum_{j=1 \mid(j \neq i)}^{N} \sum_{k=1}^{t} \frac{\mathrm{CF}(i, j, k)}{(N-1) * t}
$$

where $i$ denotes the $i$ th participant, $j$ denotes the index of the $j$ th co-player, $N$ denotes the total number of participants, and $t$ denotes the number of games in the experiments. The CF (coordination function) is defined as follows:
The SR for each of the three game strategies can then be calculated as follows:

$$
\mathrm{SR}(i)_{\{\mathrm{Acc}, \mathrm{Equ}, \mathrm{Clo}\}}=\frac{\sum_{k=1}^{14}\left[\mathrm{GT}(i, k)_{\{\mathrm{Acc}, \mathrm{Equ}, \mathrm{Clo}\}}=1\right]}{\sum_{k=1}^{14}\left|\mathrm{GT}(i, k)_{\{\mathrm{Acc}, \mathrm{Equ}, \mathrm{Clo}\}}\right|} .
$$

Together, the strategic profile of each individual player is a vector composed of three elements, one for each of the selection rules [(Acc, Equ, Clo)] using Eq. 5. For player \#7, for example, the number of games in which each of the strategies (Closeness, Accession, and Equality) was applicable was $8,10,7$, respectively, and the number of games in which each of the strategies was implemented was 5, 4, and 4, respectively. Hence, the corresponding obtained SR values were as follows: $5 / 8=0.63 ; 4 / 10=0.40 ; 4 / 7=0.57$.

\section{Results}

Before examination of the results pertaining to the $\mathrm{iCA}$ scores and the strategic profiles, it is important to demonstrate that the players were motivated to coordinate with the other unknown partner, that is, that a solution in each of the games was not randomly selected. Table 1

$$
\mathrm{CF}(i, j, k)=\left\{\begin{array}{l}
1 ; \text { if players } i \text { and } j \text { chose the same label in game } k \\
0 ; \text { otherwise. }
\end{array}\right.
$$

The iCA measure is not intended to be an absolute score, but rather it allows ranking the participants that completed the same set of tasks based on their iCA values as was the case in our study.

\subsubsection{Strategy rate (SR)}

The strategy profile is described by the weighted combination of the selection rules. To measure the frequency compares the observed CI scores with the hypothetical CI scores that would have been obtained had all the players randomly picked their solution, as expected by game theory. We can see that all decision problems were associated with a significantly higher $\mathrm{CI}$ values than random picking (a higher score denotes a better ability to coordinate). We can also notice that the difficulty of coordination varies across the different decision problems, as 


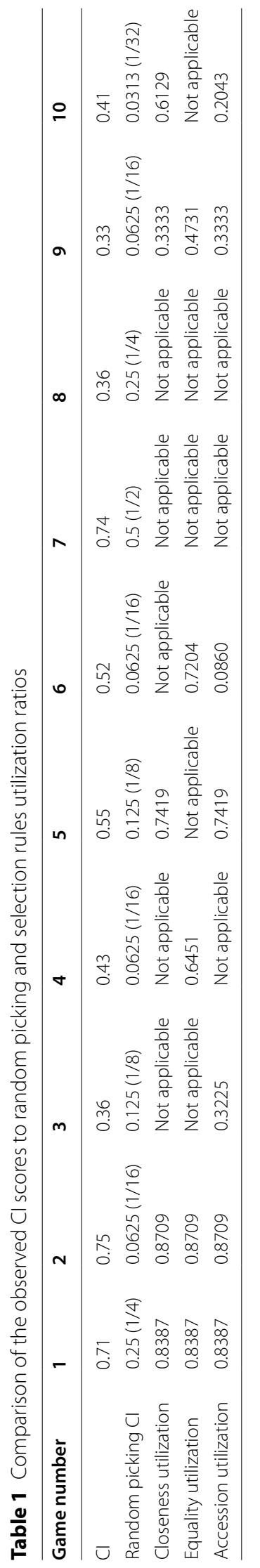




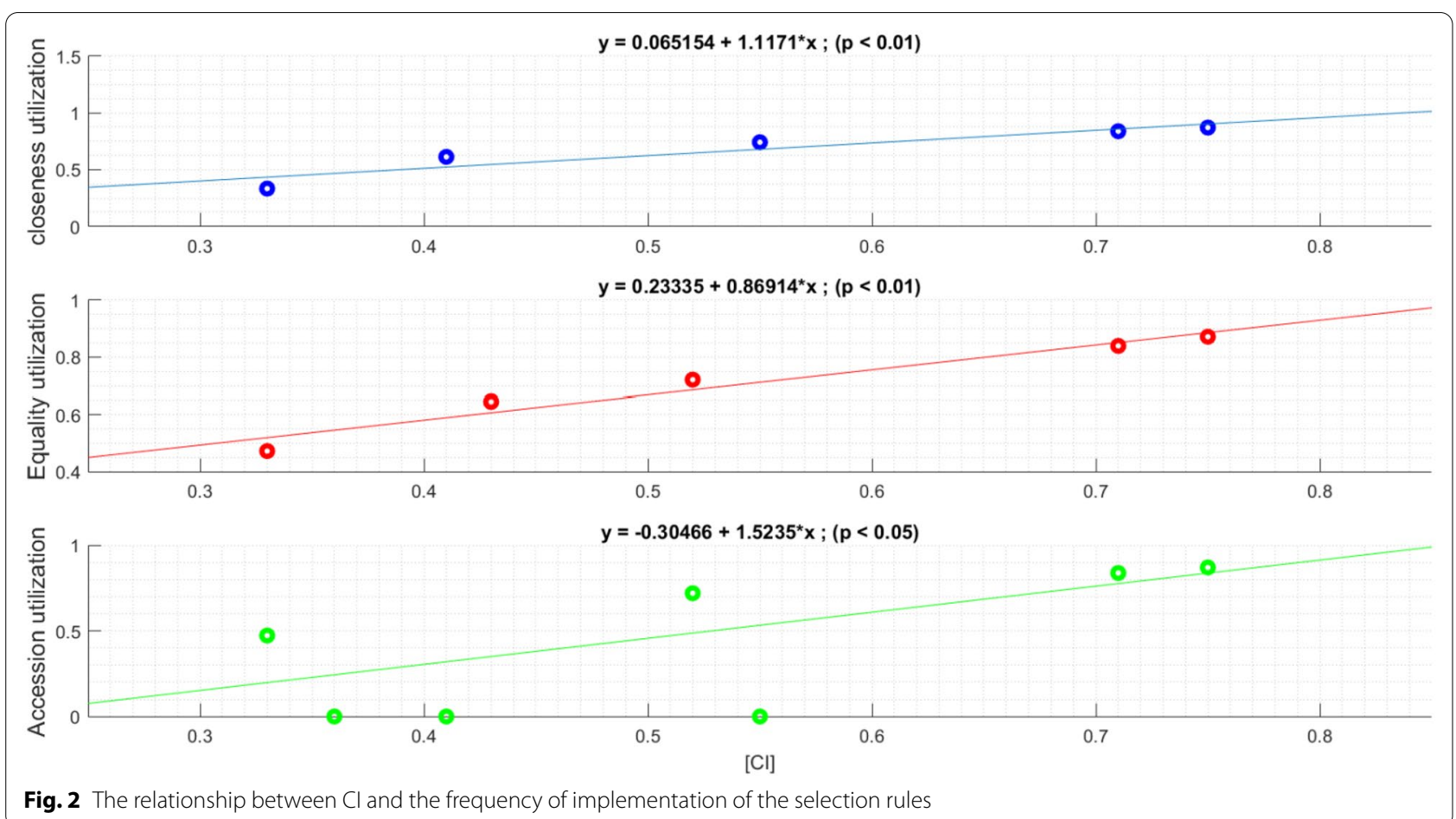

reflected by the fluctuations in the CI score. There are few easy coordination problems (e.g., \#1, \#2), few hard problems (e.g., \#3, \#8) and few problems with a medium difficulty level (e.g., \#4, \#5). Recall that the ICA is a relative measure and is calculated by pairing each player with each of the other players in each coordination problem. Therefore, all players have encountered the exact same set of predefined problems (1-10).

Figure 2 shows a regression analysis of the data shown in Table 1. Figure 2 shows that there is a positive relationship $(p<0.05)$ between the three main selection rules and the level of difficulty of the game, which is measured by the CI value. The higher the CI the easier is the game and the percentage of selection rule implementation.

\subsection{Characterizing the variability in iCA scores}

To characterize the variability in the coordination ability among different players, we have plotted the distribution of the data using a violin plot combined with boxplot, a histogram and a Gaussian mixture model (GMM) (Fig. 3A-C, respectively). It can be observed that the range of the iCA score is very diverse: the lowest score was 0.023 (average of $2 \%$ successful coordination) and the highest score 0.666 (average of $66 \%$ successful coordination). Also, we can see that the range of the iCA score was smaller in the case of the top players $(0.55,0.7)$ and therefore this group was more homogeneous compared to the lower quartile. It is also evident that the distribution is negatively skewed and that the lower $25 \%$ iCA scores are more spread out than the higher scores (Fig. 3A). In addition, the Dip test of unimodality [25] indicated that the distribution is multimodal $(p>0.1$, Dip $=0.025)$ while by estimating the distribution by a GMM with K Gaussians it was demonstrated that the entire distribution can be described by three Gaussians ( $k=3$ was determined by using Residual sum of squares). The mathematical formulation of the iCA mixture model, which is presented graphically in Fig. 3C, can be described using the following formula:

$$
\begin{aligned}
\mathrm{iCA}(x)= & 8.903^{*} \mathrm{e}^{-\left(\frac{(x-0.659)}{0.019}\right)^{2}}+5.572^{*} \mathrm{e}^{-\left(\frac{(x-0.595)}{0.023}\right)^{2}} \\
& +1.635 * \mathrm{e}^{-\left(\frac{(x-0.510)}{0.224}\right)^{2}} .
\end{aligned}
$$

It is noteworthy that this division into $k=3$ was also corroborated using the silhouette index [26] for $K$ values at the range $[1,10]$. The three Gaussians composing the distribution (Fig. 3C) comprise the third Gaussian with the lowest iCA scores, which corresponds with the lower $25 \%$, the second Gaussian which surrounds the median, and the first Gaussian which corresponds with the upper portion of the IQR as well as with the upper quartile (Fig. 3A, B). 


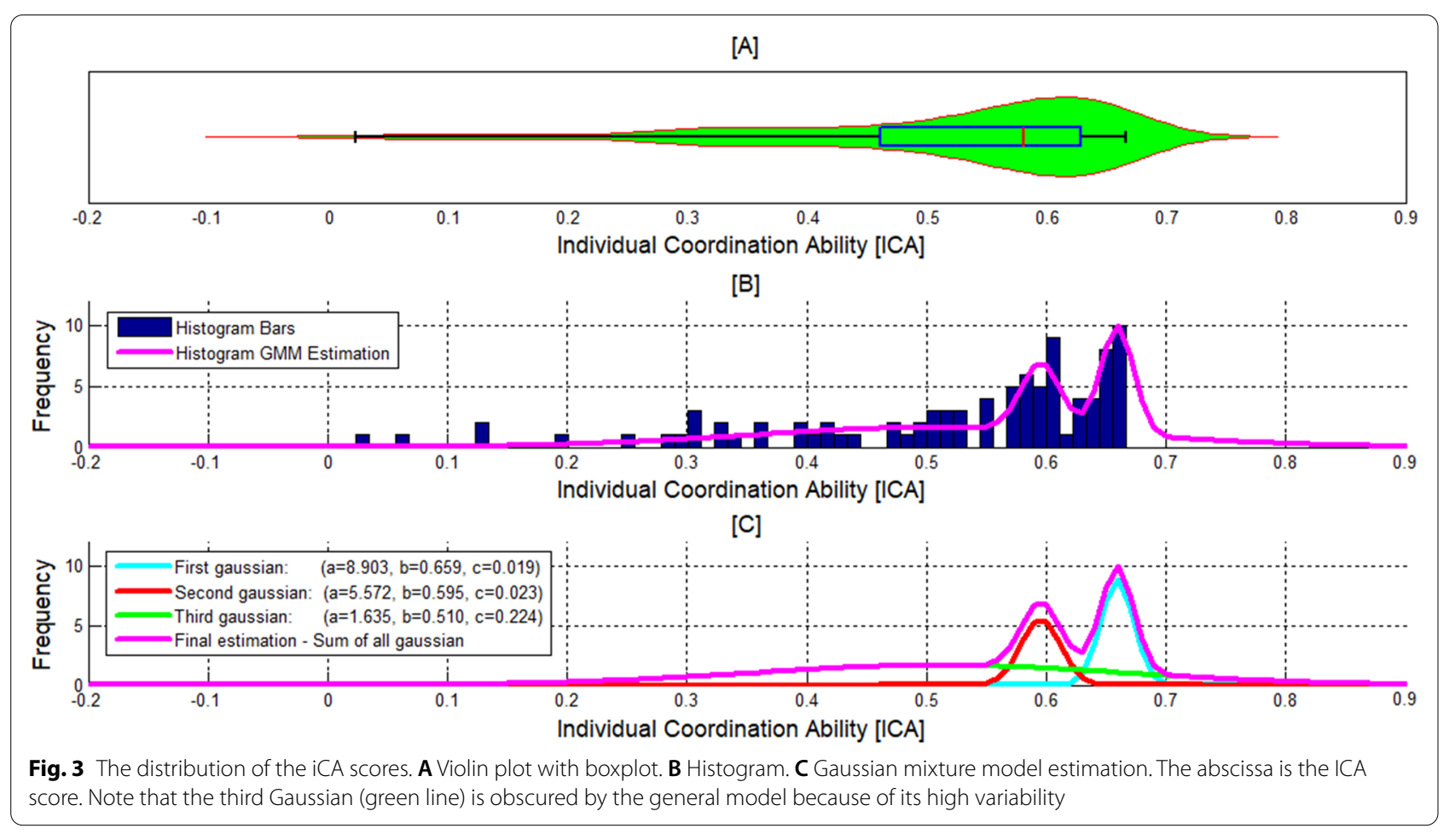

Table 2 Strategy rate statistics

\begin{tabular}{llll}
\hline & $\mathbf{S R}_{\text {Closeness }}$ & $\mathbf{S R}_{\text {Equality }}$ & $\mathbf{S R}_{\text {Accession }}$ \\
\hline Mean & 0.6919 & 0.6685 & 0.3439 \\
Standard deviation & 0.2690 & 0.2624 & 0.1569 \\
Median & 0.7500 & 0.7143 & 0.3636 \\
\hline
\end{tabular}

\subsection{Detection of dominant SR values in the strategic profile}

Before analyzing the relationship between iCA and the strategic profile, it is first necessary to identify the predominant selection rules utilized by each of the players. Table 2 displays the distribution parameters of each of the three SR indices:

It is clear from Table 2 that there were two leading selection rules: closeness, and equality. The median probability of using each of the two selection rules by a random player was over $70 \%$, if they were applicable in the game. In contrast, the accession rule had a much lower probability of selection on average by a random player.

A one-way analysis of variance showed that the main effect of strategy rate was significant $[F(2,90)=63.67$, $p<0.001$ ]. Post hoc testing (Tukey HSD) [27] indicated that the average mean value of the accession $\mathrm{SR}$ value was significantly lower than each of the other two SR values, closeness, and equality $(p<0.001)$.

\subsection{Modeling the relationship between strategic profile and iCA}

The previous section showed that the strategic profile contains two leading strategies (closeness and equality) and another secondary strategy (accession). This phenomenon may affect the performance of the predictive model due to unwanted dependencies between the independent variables $\left(\mathrm{SR}_{\text {Closeness }}, \mathrm{SR}_{\text {Equality }}, \mathrm{SR}_{\text {Accession }}\right)$. On the other hand, the omission of one of these variables results in discarded data that may consequently lead to impaired performance of the predictive model. To deal with this problem we will use a dimensionality reduction technique [28-30] to maximize the variance of the data in a smaller number of features, thereby reducing the dependencies among the variables. To do so we used the principal component analysis (PCA) algorithm [29]. We have first conducted feature selection and then performed feature extraction, i.e., we have constructed a new feature space by data compression [29, 31].

For feature selection we have first extracted the new base vectors, namely, the principal components. The components were extracted by calculating the eigenvectors of the covariance matrix of the data after conducting mean and variance normalization. Then, the principal components were sorted according to the magnitude of their coefficients (i.e., eigenvectors) from largest to 


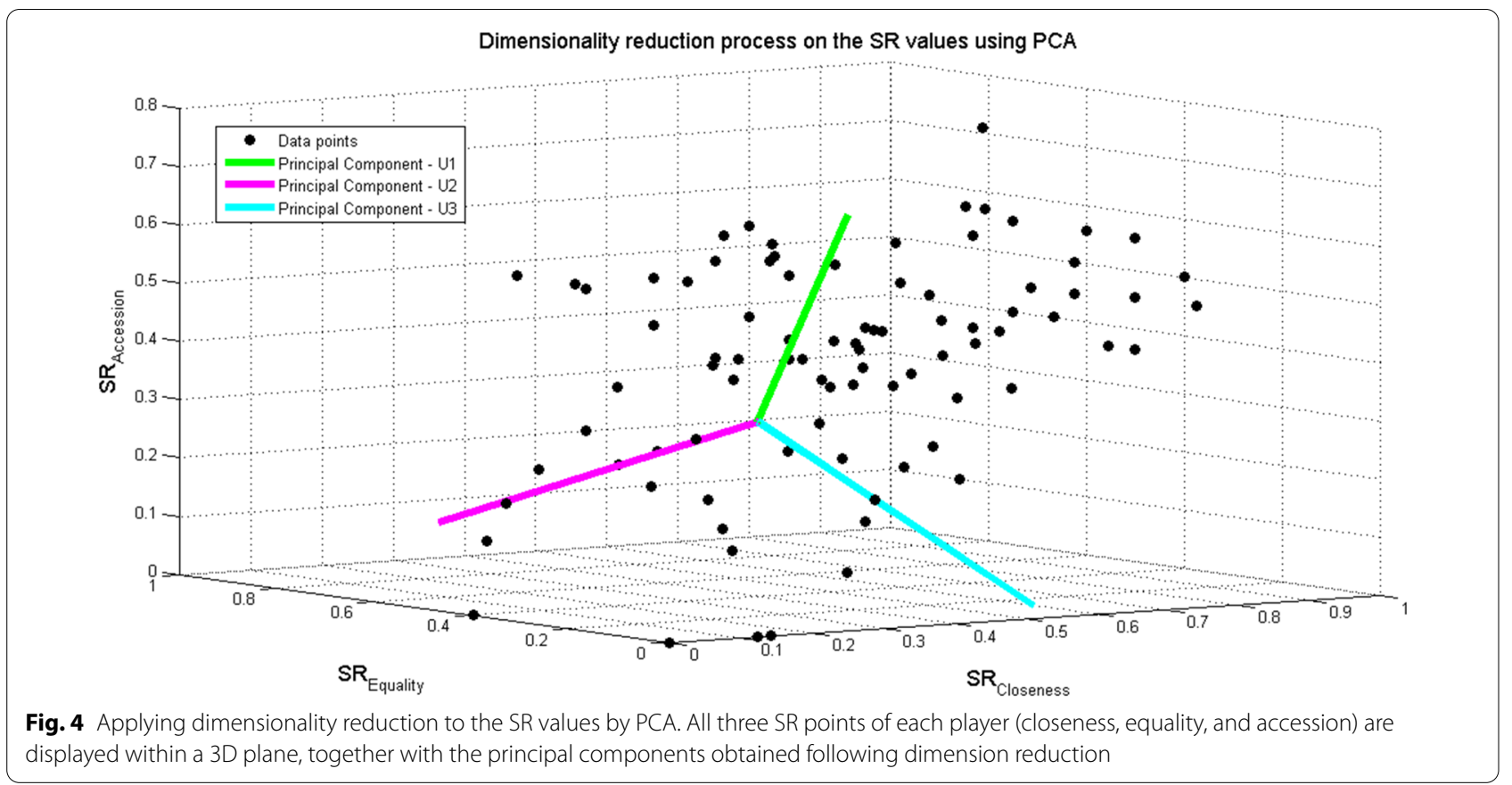

Table 3 Retained variance in dimension reduction process

\begin{tabular}{llll}
\hline Number of principal components & $\mathbf{1}$ & $\mathbf{2}$ & $\mathbf{3}$ \\
\hline Retained variance (\%) & 70.74 & 90.43 & 100 \\
\hline
\end{tabular}

smallest (in absolute values). Figure 4 presents the three principal components (each denoted by $U$ ) overlaid on the original data (based on the three SR values). Next, we performed feature extraction and selected the two components, $U 1$ and $U 2$ with the highest explained variance (i.e., with the two largest eigenvalues) to create a new dimensional feature space. Consequently, we have projected the original data onto a new feature space while retaining the maximum variance in the original dataset (Table 3). Thus, we have compressed the original 3D data into a new 2D space. Specifically, two new data features have been produced: $S_{1}$-the projection of the vector $x$ on $U 1$, and $S_{2}$-the projection of the vector $x$ on $U 2$.

To ensure that no critical data were lost during the process of dimensionality reduction, we performed a reconstruction of the reduced data into the original dimension while comparing the reconstructed data to the original data vector before compression. The strategic profile of the $i$ th player is denoted by $x^{(i)}$ vector, which contains the three SR values. The retained variance calculation was performed as follows:

$$
\text { Retained variance }=1-\frac{\frac{1}{m} \sum_{i=1}^{m}\left\|x^{(i)}-x_{\text {approx }}^{(i)}\right\|^{2}}{\frac{1}{m} \sum_{i=1}^{m}\left\|x^{(i)}\right\|^{2}} .
$$

The calculation for each number of selected principal components produced the following results, described in Table 3.

As can be seen in Table 3, for two principal components new data vectors can be produced that contain about $90 \%$ of the variance of the original data, where the correlation between the two variables is negligible (due to the dimensionality reduction process).

Following data compression, we performed a regression with the single dependent variable being the coordination ability of the $i$ th player, described by the iCA score, while the predictors were the newly found $S 1$ and $S 2$ features. In order to find the model coefficients, we used multiple linear regressions:

$$
\operatorname{ICA}(i)=0.52118+0.090788 * S_{1}+0.054041 * S_{2},
$$

$$
R^{2}=0.8733 ; F=310.0768 ; p<0.001 ; \operatorname{VAR}_{\text {error }}=0.0029,
$$

where

$$
\begin{aligned}
S 1= & 0.610 * \mathrm{SR}_{\text {Closeness }} \\
& +0.518 * \mathrm{SR}_{\text {Equality }} \\
& +0.599 * \mathrm{SR}_{\text {Accession }},
\end{aligned}
$$




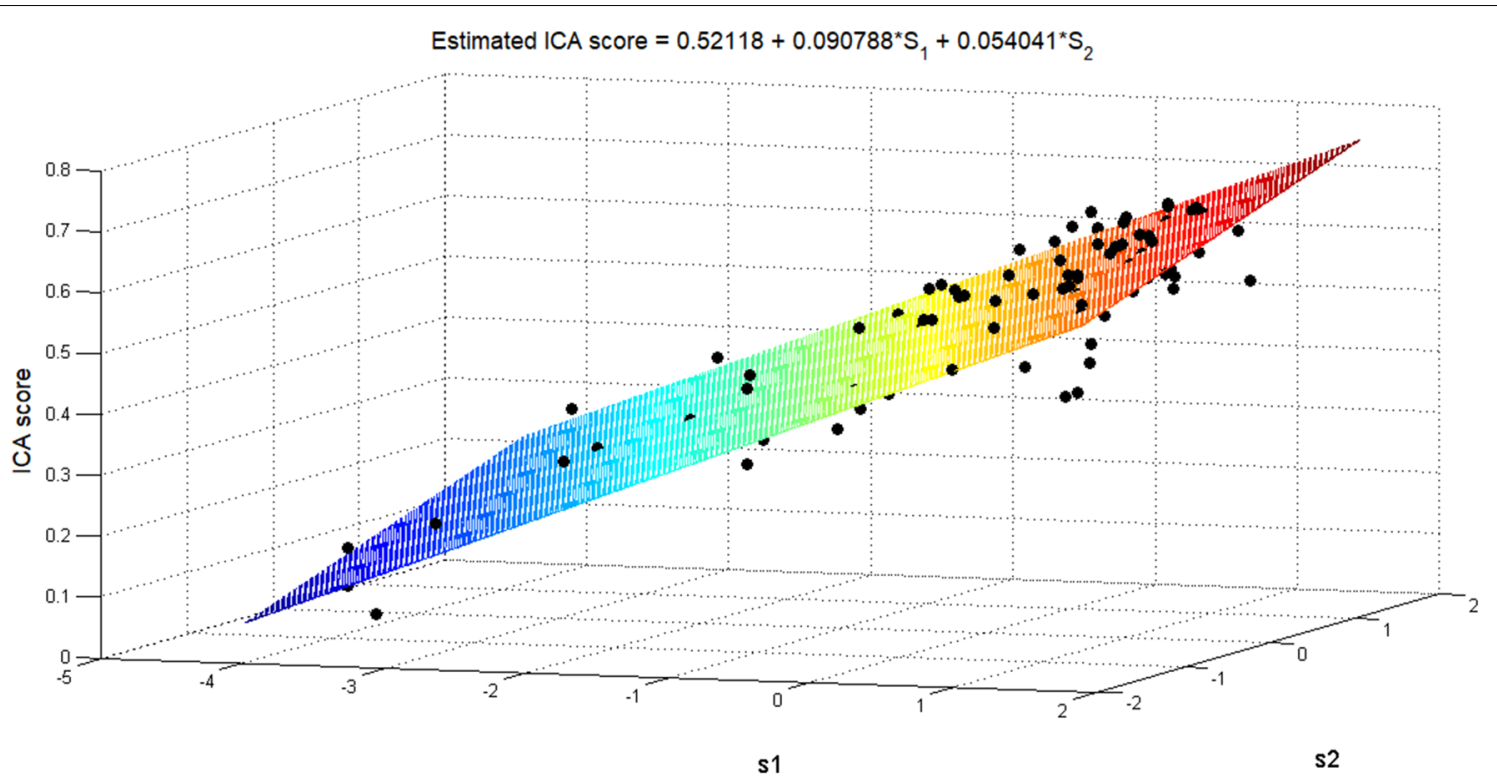

Fig. 5 The regression model in a 3D space. A multi-variable regression model that describes the relationship between the three SR variables and iCA. Each dot represents the iCA value of each player as a function of their strategic profile after data compression (3D to 2D). The new model is presented as a $2 \mathrm{D}$ plane in a 3D space

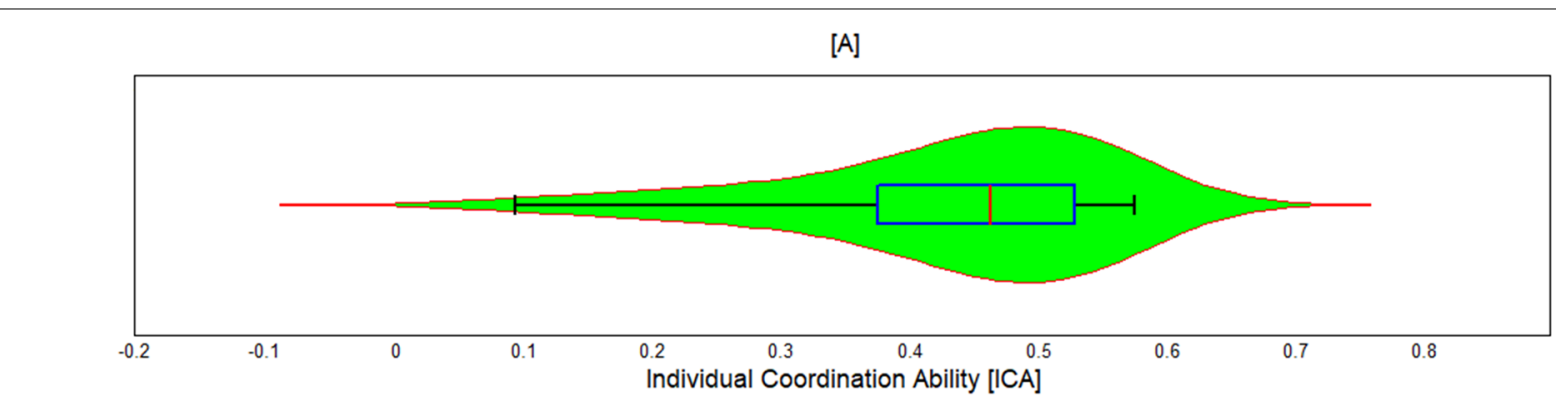

[B]

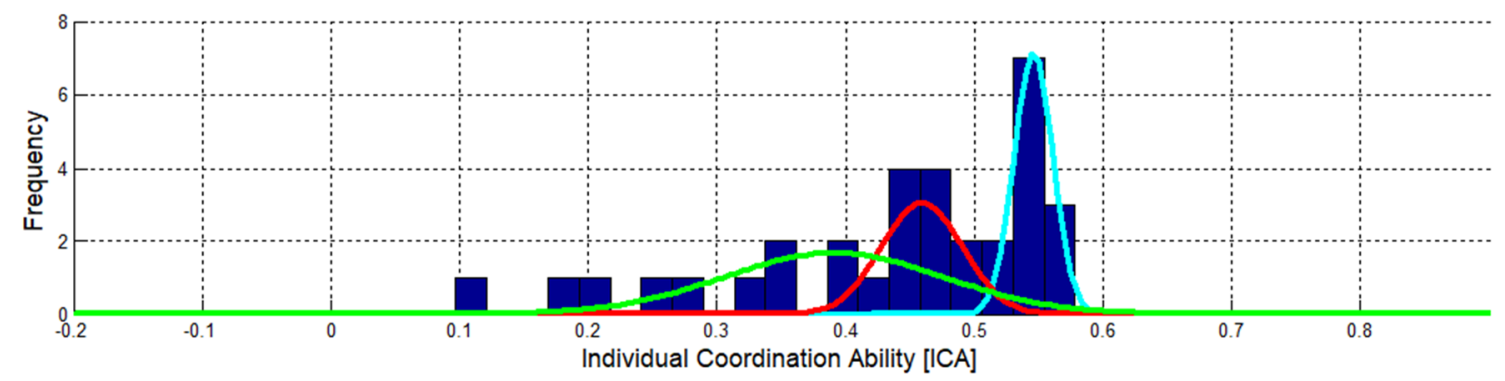

Fig. 6 ICA score distribution of the validation group. A Violin plot with boxplot. B Histogram with Gaussian mixture model estimation 
Table 4 Percentiles of the iCA relative absolute error distribution

\begin{tabular}{lll}
\hline 25th percentile & 50th percentile (median) & 75th percentile \\
\hline 3.915 & 7.811 & 13.314
\end{tabular}

$$
\begin{aligned}
S 2= & -0.312 * \mathrm{SR}_{\text {Closeness }}+0.853 * \mathrm{SR}_{\text {Equality }} \\
& +-0.418 * \mathrm{SR}_{\text {Accession }} .
\end{aligned}
$$

We can see from Eq. 8.2 the strong correlation between iCA and the strategic profile, while our correlation is based only on two variables, $S_{1}$ and $S_{2}$. This allows us to draw a 2D plane, which represents the model, in a threedimensional space overlaid on the corresponding iCA values, as can be seen in Fig. 5.

\subsection{Model validation}

In the previous section, we presented a model (Eqs. 8.1, $8.2,8.3,8.4)$ that links between the iCA scores and the individual strategic profiles of the players. In this section, we present validation results to assure that the model can be applied on a new dataset that was not used for model construction. Here, we demonstrate the robustness of the model by predicting the iCA values of players based on their individual strategic profile.

\subsubsection{Participants}

The participants in the validation group were 33 students that were enrolled in one of the courses on campus [ 12 of whom were female, mean age $=24.23$ (years), $\mathrm{SD}=2.21]$. The validation group participated in the same experimental design as the original group (see Sects. 2.1 and 2.2).

\subsection{2 iCA distribution}

Figure 6 displays the iCA score distribution of the validation group. It can be seen that the $\mathrm{iCA}$ distribution of the two groups (original group and validation group) is very similar (compare Fig. 6 with Fig. 3). To validate this apparent similarity, the iCA distribution of the validation group was estimated by a GMM. It was demonstrated that the validation group was characterized with the same iCA distribution as the original study group. That is, the entire distribution of iCA scores could also be described by three Gaussians $(k=3)$ and a similar partitioning into sub-groups according to the level of coordination ability was observed (Fig. 6A, B). That is, there is a group of players with a high coordination ability (blue Gaussian, coinciding with the upper quartile), a group of players with an intermediate coordination ability (red Gaussian, surrounding the median) and a group of players with a low coordination ability that manifests a wide dispersion (green Gaussian, coinciding with the lower portion of the IQR and the lower 25\%).

\subsubsection{Prediction of the iCA score and model evaluation}

To assess the accuracy of the proposed prediction model (Eq. 8.1) we have examined the statistical indices of the relative error between the predicted value estimated by the model and the real value calculated using the iCA formula (Eq. 2). The relative absolute error is defined as follows:

$$
\begin{aligned}
& \mathrm{iCA} \text { relative absolute error }(\%) \\
& =\frac{\left|\mathrm{iCA}_{\text {real }}-\mathrm{iCA} \mathrm{A}_{\text {predicted }}\right|}{\mathrm{iCA} \text { real }} * 100 .
\end{aligned}
$$

Table 4 presents the percentiles of the distribution of the ICA relative absolute error. It can be observed that the median relative absolute error was only $7.811 \%$ while $75 \%$ of the total population does not exceed an error of $13.314 \%$. In addition, out of 33 predictions, there were only 3 values for which the relative absolute error was greater than $30 \%$.

These results demonstrate that the predictive model is robust and can be generalized to an independent dataset. That is, the estimated iCA values of a player can be predicted based on their strategic profile with high accuracy.

\section{Discussion}

This research contributes to key issues pertaining to the process of decision-making in tacit coordination. First, although in previous studies the variability among players has been examined in different domains (e.g., [18, $32])$, in our study the variability was explored at the individual level of analysis in the domain of tacit coordination games. Second, to find the predominant selection rules that were applied during task performance, we devised the SR index that was computed for each individual player. This analysis hinted to the existence of two predominant selection rules: closeness and equality. This indicates that certain selection rules are preferred by players and are more useful for coordination than others in the context of a specific task. Third, we devised a novel method for constructing a strategic profile for each individual player. The method relies on the projection of the individual SR indices onto a 3D strategy space. Finally, we have used multiple regression analysis and showed that there is a strong correlation between the player's strategic profile and their coordination ability.

Previous research dealing with focal points examined a wide range of selection rules in different games (e.g., $[12,13,18,33])$. In these previous studies, the data were processed and analyzed at the group (aggregate) level only, and so no relationship was demonstrated between 
individual coordination ability and the set of strategies implemented during the game as was done here. Yet in several other studies $[15,16,20]$ an individual level of analysis has been implemented. However, in these studies the emphasis was on examining the effect of social factors (e.g., culture [15], social value orientation [20, 34] and loss aversion [35]) on strategic behavior whereas the current study is more generic as it does not utilize any prior knowledge about the players.

Importantly, while our work shows the ability to model individual coordination ability and use it to predict behavior, our framework is demonstrated by using only one domain and its generalizability is yet to be explored. For example, given a new coordination domain, the selection rules have to be defined first and only then our model could be applied. The preprocessing step of finding the selection rules in a given domain is still lacking in the absence of a formal procedure for extracting the selection rules.

Thus, the main contribution of this study is the construction of a model predicting individual coordination ability although this dependent variable was characterized by a multimodal distribution (see Figs. 3,5 ). This multimodal distribution might corroborate cognitive hierarchy theory [36-38], which postulates that individuals differ in their depth of reasoning. By this account, an agent is bounded by the $k$ steps of reasoning they can perform [39, 40]. Thus, each of the three Gaussians found in the current study may correspond to a different level $k$ that bounds the best response given by the players. Hence, the second and third Gaussians found in our study may each correspond to a level $k \geq 1$, while the first Gaussian probably includes a substantial number of players with a level $k=0$ (see Figs. 3C, 6B). Noteworthily, the suggested connection between our findings and level $k$ theory should be further explored especially in view of findings indicating inconsistencies in the level $k$ of players, even in the same game, which imply that the depth of reasoning is stochastic in nature $[41,42]$.

Nevertheless, our results can also be compatible with a team reasoning approach $[18,43-46]$. However, it was previously shown that coordination rate on a focal point depends on whether the game is symmetric or not as well as on stake size [47]. Therefore, the mode of reasoning of the player may be explained by either cognitive hierarchy or team reasoning, depending on specific game features [47].

The focal points that were modeled in our study are based on spatial properties (e.g., closeness, equality, and accession). Consequently, we expect the model we have constructed to be applicable to other contexts where focal points are based on spatial cues, e.g., "Bargaining Table" [14, 35, 40, 48] and "Moving Discs" [18]. However, in other cases, where focal points are based on nonspatial features (e.g., the "word Selection" task based on semantic meaning [18, 24, 42, 49]) our model should be modified accordingly. For example, by updating the number of Gaussians which correspond to different strategic profiles extracted from the game.

\section{Conclusions}

In summary, in this study we investigated the distribution of individual coordination ability in tacit coordination games and constructed a predictive model based on the individual strategic profiles of the players. The strong relationship found in our study between the strategic profile and coordination ability suggests that players differ in the strength by which they prefer salient choices [50] and this in turn might affect their coordination ability. Our findings could be explained by either cognitive hierarchy or team reasoning. Nevertheless, they indicate that individual-level analysis is essential for gaining insight into the diversity among decision-makers regarding their strategic performance.

The results of the current study open several avenues for future research. First, it will be interesting to consider tacit coordination games of divergent interests (e.g., [33, 48]). That is, games in which the parties are required to coordinate their answers, while different outcomes of the game may yield different utilities for each of the players. Second, it is worthwhile examining the effect of personality traits and attitudes that correlate with coordination ability, such as introversion-extroversion [51], or social value orientation (e.g., $[20,52,53])$, on the strategic profiles of the players. Third, improved models in the framework of human-machine coordination [3, 54] can optimize performance in diverse contexts such as intension prediction [55] and safety [56] in industry, fraud detections (e.g., [57-59]), as well as in other complex tasks (e.g., [60]). Fourth, it might also be worthwhile exploring whether the differences in individual coordination ability are accompanied by parallel changes in brain activation reflected by electrophysiological markers associated, for example, with cognitive load [24] or with time pressure [50]. These electrophysiological markers could then be added to our model as additional features that may improve its predictive ability. Finally, it is possible that strategic profiles are better described by the complexity level of the game (i.e., number of possible solutions $-2^{\# \text { number of discs }}$ ) rather than by the selection rules 


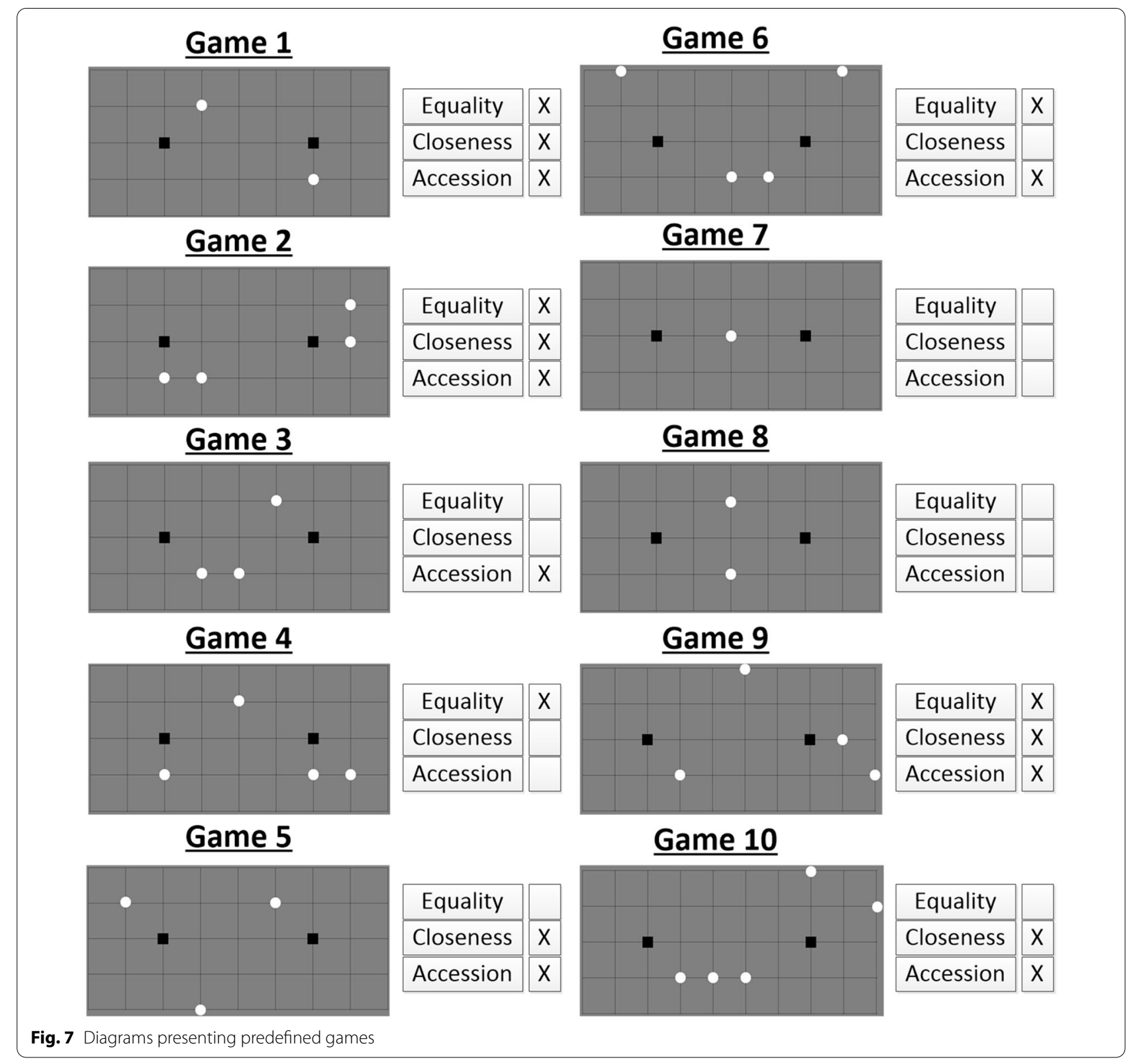

themselves. Therefore, it is worthwhile to repeat the current study while the set of games is divided into distinct levels of coordination difficulty.

\section{Appendices}

\subsection{Appendix A: "Assign Circles" predefined game boards}

The 10 predefined games are presented in Fig. 7. The board's structure is based on the design of questions 11-20 in [12]. The order of appearance of the various games in the application was completely random. Next to each game board, the availability of the three main selection rules, which is used to construct the strategic profile, is presented.

\subsection{Appendix B: Selection rules implementation in the predefined games (\#1-10)}

In this section, we present the complete definition of the three selection rules. The following definitions are directly quoted from [12], pp. 173-175.

\subsubsection{Closeness}

For the rule of closeness to be applicable in a game, there has to be some commonly recognized concept of closeness of association between the two classes of objects (circles and squares). In the case of our grid, the most obvious and unambiguous measure of closeness of 
Table 5 Implication of the main selection rules

\begin{tabular}{|c|c|c|c|c|}
\hline \multirow[t]{2}{*}{ Game } & \multicolumn{3}{|c|}{$\begin{array}{l}\text { Unique assignment implied by rule } \\
\text { of }\end{array}$} & \multirow[t]{2}{*}{ Predicted responses } \\
\hline & Closeness & Accession & Equality & \\
\hline 1 & $L R$ & $L R$ & $L R$ & $L R$ \\
\hline 2 & LLRR & LLRR & LLRR & LLRR \\
\hline 3 & None & LLR & None & LLR \\
\hline 4 & None & None & LLRR & LLRR \\
\hline 5 & LLR & LLR & None & None \\
\hline 6 & None & LRRR & LLRR & LRRR or LLRR \\
\hline 7 & None & None & None & None \\
\hline 8 & None & None & None & None \\
\hline 9 & LRRR & LRRR & LLRR & LRRR or LLRR \\
\hline 10 & LLRRR & LLLRR & None & LLRRR or LLLRR \\
\hline
\end{tabular}

association between a circle and a square is the distance between them. Thus, we interpret the rule of closeness as: assign each circle to the nearer square.

\subsubsection{Accession}

The rule of accession implies that if a set of circles form a coherent group, all the circles in the group should be assigned to the same square. We shall say that two circles are connected if they are located at adjacent points in the grid, linked by a horizontal or vertical line, and we shall interpret a 'coherent group' as a set of connected circles. We define the distance between a square and a set of connected circles as the distance between the square and the nearest circles in the set. Then we interpret the rule of accessions the following formula: assign each set of connected circles to the nearer square.

\subsubsection{Equality}

The rule of equality suggests the general formula: if there is an even number of circles, assign half of them to the one square and half of them to the other square. As stated, this rule never implies a unique assignment of circles to squares; we posit the median line rule as a subrule of refinement of the rule of equality, which uses the metric of closeness to discriminate among equal assignments. This rule is: if there is a vertical line such that an equal number of circles lie on each side, then assign circles left of the line to the left-hand square, and circles right of the line to the right-hand square.

Table 5 in Appendix (taken from [12]) presents the specific solutions obtained by implementing each one of the three selection rules in games 1-10. Each one of the selection rules can only be implemented in a game board only if it defines a unique choice (there is only a single interpretation of the solution). In Table 5 "L" represents a connection of a circle to the left square and
" $R$ " to the right square. The circles are ordered from left to right and from top to bottom. For example, in game \#3 the assignment implied by the rule of accession denoted by "LLR", means that the two bottom circles are connected to the left square and that the upper circle is connected to the right square.

Acknowledgements

Not applicable.

Authors' contributions

All authors (DM, IL and IZ) carried out the stages of conceptualization,

design of methodology, data curation, formal analysis, data modeling, model validation, writing and editing. DM was also responsible for visualization and implementation of supporting algorithms. IL and IZ supervised the research activity. All authors discussed the results. All authors read and approved the final manuscript.

Funding

The authors received no specific funding for this work.

\section{Availability of data and materials}

The datasets generated and/or analyzed during the current study, together with the corresponding analysis codes (which fits MATLAB R2016a) are available on the website of the "NeurolS Lab" at Ariel University, (https://www.ariel. ac.il/wp/neurois/).

\section{Declarations}

Ethics approval and consent to participate

The study was approved by the IRB committee of Ariel University. All participants provided written informed consent.

\section{Consent for publication}

All authors (DM, IL and IZ) consent to the publication of the manuscript, if editorially accepted for publication, in Brain Informatics, published by Springer.

\section{Competing interests}

The authors declare that they have no competing interests.

Received: 7 May 2020 Accepted: 13 January 2022

Published online: 04 February 2022

\section{References}

1. Bacharach M (2006) Beyond individual choice: teams and frames in game theory. Wiley, Hoboken

2. Mehta J (1997) Telling tales: actors' accounts of their behavior in coordination games. The 17th Arne Ryde Symposium, "Focal Points-Coordination, Complexity, and Communication in Strategic Contexts," Lund

3. Fenster M, Kraus S, Rosenschein JS (1995) Coordination without communication: experimental validation of focal point techniques. Proceedings of the First International Conference on Multi-Agent Systems. AAAI Press, California, pp 102-108

4. Schelling TC (1960) The strategy of conflict. Harvard University, Cambridge

5. Binmore K (2007) Game theory: a very short introduction. Oxford University Press, Oxford

6. Ezeala-Harrison F, Baffoe-Bonnie J (2016) Market concentration in the grocery retail industry: application of the basic prisoners' dilemma model. Appl Econ 6:47

7. Fang TP, Wu A, Clough DR (2021) Platform diffusion at temporary gatherings: social coordination and ecosystem emergence. Strateg Manag J 42:233-272

8. Gansterer M, Hartl RF (2021) The prisoners' dilemma in collaborative carriers' request selection. Cent Eur J Oper Res 29:73-87

9. Larrouy $L$ (2015) Revisiting methodological individualism in game theory: the contributions of Schelling and Bacharach. Université Côte d'Azur, France 
10. Gilbert M (1989) Rationality and salience. Philos Stud 57:61-77

11. Colman AM (1997) Salience and focusing in pure coordination games. J Econ Methodol 4:61-81

12. Mehta J, Starmer C, Sugden R (1994) Focal points in pure coordination games: an experimental investigation. Theory Decis 36:163-185

13. Mehta J, Starmer C, Sugden R (1994) The nature of salience: an experimental investigation of pure coordination games. Am Econ Rev 84:658-673

14. Mizrahi D, Laufer I, Zuckerman I (2021) Predicting focal point solution in divergent interest tacit coordination games. J Exp Theor Artif Intell. https://doi. org/10.1080/0952813X.2021.1974953

15. Mizrahi D, Laufer I, Zuckerman I (2020) Collectivism-individualism: strategic behavior in tacit coordination games. PLoS ONE. https://doi.org/10.1371/journ al.pone.0226929

16. Mizrahi D, Laufer I, Zuckerman I (2020) Individual strategic profiles in tacit coordination games. J Exp Theor Artif Intell 33:1-16

17. Mizrahi D, Laufer I, Zuckerman I (2019) Modeling individual tacit coordination abilities. International conference on brain informatics. Springer, Cham, pp 29-38

18. Bardsley N, Mehta J, Starmer C, Sugden R (2009) Explaining focal points: cognitive hierarchy theory versus team reasoning. Econ J 120:40-79

19. Crawford VP (1985) Learning behavior and mixed-strategy Nash equilibria. J Econ Behav Organ 6:69-78. https://doi.org/10.1016/0167-2681(85)90025-3

20. Mizrahi D, Laufer I, Zuckerman I, Zhang T (2018) The effect of culture and social orientation on player's performances in tacit coordination games. International Conference on Brain Informatics. Springer, Cham, pp 437-447

21. Colman AM, Pulford BD, Lawrence CL (2014) Explaining strategic coordination: cognitive hierarchy theory, strong Stackelberg reasoning, and team reasoning. Decision 1:35-58

22. Misyak JB, Chater N (2014) Virtual bargaining: a theory of social decisionmaking. Philos Trans R Soc B Biol Sci. https://doi.org/10.1098/rstb.2013.0487

23. Pulford BD, Colman AM, Buabang EK, Krockow EM (2018) The persuasive power of knowledge: testing the confidence heuristic. J Exp Psychol Gen 147:1431-1444

24. Mizrahi D, Laufer I, Zuckerman I (2020) The effect of individual coordination ability on cognitive-load in tacit coordination games. In: Davis F, Riedl R, vom Brocke J et al (eds) NeurolS Retreat 2020. Springer, Vienna

25. Hartigan JA, Hartigan PM (1985) The dip test of unimodality. Ann Stat 13:70-84

26. Rousseeuw PJ (1987) Silhouettes: a graphical aid to the interpretation and validation of cluster analysis. J Comput Appl Math 20:53-65

27. Tukey JW (1949) Comparing individual means in the analysis of variance. Biometrics 5:99-114

28. Yan S, Xu D, Zhang B et al (2007) Graph embedding and extensions: a general framework for dimensionality reduction. IEEE Trans Pattern Anal Mach Intell. https://doi.org/10.1109/TPAMI.2007.250598

29. van der Maaten L, Postma E, van den Herik J (2009) Dimensionality reduction: a comparative review. J Mach Learn Res 10:66-71

30. Lee JA, Verleysen M (2007) Nonlinear dimensionality reduction. Springer Science \& Business Media, Berlin

31. Hoffmann H (2007) Kernel PCA for novelty detection. Pattern Recognit 40:863-874. https://doi.org/10.1016/j.patcog.2006.07.009

32. Hargreaves Heap SP, Rojo Arjona D, Sugden R (2017) Coordination when there are restricted and unrestricted options. Theory Decis 83:107-129

33. Isoni A, Poulsen A, Sugden R, Tsutsui K (2013) Focal points in tacit bargaining problems: experimental evidence. Eur Econ Rev 59:167-188

34. Mizrahi D, Laufer I, Zuckerman I (2021) The effect of expected revenue proportion and social value orientation index on players' behavior in divergent interest tacit coordination games. International Conference on Brain Informatics. Springer, Cham, pp 25-34

35. Mizrahi D, Laufer I, Zuckerman I (2020) The effect of loss-aversion on strategic behaviour of players in divergent interest tacit coordination games. International Conference on Brain Informatics. Springer, Cham, pp 41-49

36. Bacharach M, Stahl DO (2000) Variable-frame level-n theory. Games Econ Behav 32:220-246

37. Camerer C, Ho T-H, Chong K (2004) A cognitive hierarchy model of games. Q J Econ 119:861-898

38. Stahl DO, Wilson PW (1995) On players' models of other players: theory and experimental evidence. Games Econ Behav 10:218-254

39. Strzalecki T (2014) Depth of reasoning and higher order beliefs. J Econ Behav Organ 108:108-122
40. Mizrahi D, Zuckerman I, Laufer I (2020) Using a stochastic agent model to optimize performance in divergent interest tacit coordination games. Sensors 20:7026

41. Cooper DJ, Fatas E, Morales AJ, Qi S (2016) Consistent depth of reasoning in level-k models. Universidad de Málaga, Málaga

42. Mizrahi D, Laufer I, Zuckerman I (2021) Level-k classification from EEG signals using transfer learning. Sensors 21:7908

43. Colman AM, Gold N (2018) Team reasoning: solving the puzzle of coordination. Psychon Bull Rev 25:1770-1783

44. Faillo M, Smerilli A, Sugden R (2013) The roles of level-k and team reasoning in solving coordination games. Cognitive and Experimental Economics Laboratory, Trento

45. Gold N, Colman AM (2018) Team reasoning and the rational choice of payoff-dominant outcomes in games. Topoi. https://doi.org/10.1007/ s11245-018-9575-z

46. Bacharach M (1999) Interactive team reasoning: a contribution to the theory of cooperation. Res Econ 53:117-147

47. Parravano M, Poulsen O (2015) Stake size and the power of focal points in coordination games: experimental evidence. Games Econ Behav 94:191-199

48. Isoni A, Poulsen A, Sugden R, Tsutsui K (2019) Focal points and payoff information in tacit bargaining. Games Econ Behav 114:193-214

49. Mizrahi D, Laufer I, Zuckerman I (2021) Topographic analysis of cognitive load in tacit coordination games based on electrophysiological measurements. NeurolS retreat 2021. Springer, Vienna

50. Li X, Camerer C (2019) Using visual salience in empirical game theory. SSRN. https://doi.org/10.2139/ssrn.3308886

51. Eysenck H (2017) The biological basis of personality. Routledge, New York

52. Murphy RO, Ackermann KA, Handgraaf MJJ (2011) Measuring social value orientation. Judgm Decis Mak 6:771-781

53. Zuckerman I, Cheng KL, Nau DS (2018) Modeling agent's preferences by its designer's social value orientation. J Exp Theor Artif Intell 30:257-277. https://doi.org/10.1080/0952813X.2018.1430856

54. Zuckerman I, Kraus S, Rosenschein JS (2011) Using focal point learning to improve human-machine tacit coordination. Auton Agent Multi Agent Syst 22:289-316

55. Wang W, Li R, Chen Y, Jia Y (2018) Human intention prediction in humanrobot collaborative tasks. ACM/IEEE International Conference on HumanRobot Interaction, Japan, pp 279-280

56. Bausys R, Cavallaro F, Semenas R (2019) Application of sustainability principles for harsh environment exploration by autonomous robot. Sustainability 11:1-18

57. Srivastava A, Kundu A, Sural S, Majumdar AK (2008) Credit card fraud detection using Hidden Markov Model. IEEE Trans Dependable Secur Comput 5:37-48

58. Bolton RJ, Hand DJ (2002) Statistical fraud detection: a review. Stat Sci. https://doi.org/10.1214/ss/1042727940

59. Johnson PE, Grazioli S, Jamal K (1993) Fraud detection: intentionality and deception in cognition. Account Organ Soc 18:467-488. https://doi.org/ 10.1016/0361-3682(93)90042-5

60. Semenas R, Bausys R (2020) Modelling of autonomous search and rescue missions by interval-valued neutrosophic WASPAS framework. Symmetry 12:162. https://doi.org/10.3390/sym12010162

61. Binmore K (2007) Playing for real: a text on game theory. Oxford University Press, Oxford

\section{Publisher's Note}

Springer Nature remains neutral with regard to jurisdictional claims in published maps and institutional affiliations. 